\title{
Educação interprofissional e o Programa de Educação pelo Trabalho para a Saúde/Rede Cegonha:
}

\author{
potencializando mudanças na formação acadêmica
}

\author{
Franklin Delano Soares Forte(a) \\ Hannah Gil de Farias Morais ${ }^{(b)}$ \\ Shirley Arruda Guimarães Rodrigues ${ }^{(c)}$ \\ Joyce da Silva Santos(d) \\ Priscila Farias de Albuquerque Oliveira ${ }^{(e)}$ \\ Maria do Socorro Trindade Morais ${ }^{(f)}$ \\ Talitha Emanuelle Barbosa Galdino de Lira(g) \\ Maria de Fátima Moraes Carvalho(h)
}

\section{Introdução}

Nos últimos anos, observou-se que as práticas interprofissionais despertaram bastante interesse, tanto no governo como nas instituições de Ensino Superior formadoras dos profissionais de saúde. Algumas pesquisas sugerem que a colaboração interprofissional, tanto na estratégia usuário-centrada como no aprendizado baseado em equipe, favorece, sinergicamente, práticas mais significativas para a produção do cuidado em saúde ${ }^{1,2}$. Sabe-se da importância da colaboração de diversas áreas para o cuidado em saúde. A educação interprofissional mostrou-se uma estratégia importante para a promoção desse objetivo ${ }^{3}$.

A perspectiva compreendida de educação interprofissional se dá "quando estudantes ou profissionais de dois ou mais cursos ou núcleos profissionais aprendem sobre os outros, com os outros e entre si" 4 (p. 7). Esse movimento de aprendizado possibilita uma colaboração eficaz e melhora os resultados na saúde ${ }^{4}$. Assim, neste texto, a interprofissionalidade foi usada ${ }^{2}$ com foco: nas relações interprofissionais, no Programa de Educação pelo Trabalho da Rede de Atenção à Saúde Materno-Infantil, a Rede Cegonha.

As complexidades do mundo e da cultura exigem análises mais integradas. Qualquer acontecimento humano apresenta diversas dimensões, uma vez que a realidade é multifacetada. É neste contexto que se coloca a interprofissionalidade, que, ao invés de se apresentar como alternativa para a substituição de um jeito de produzir e transmitir conhecimento, se propõe a ampliar as visões de mundo, de nós mesmos e da realidade, com o propósito de superar a visão disciplinar 5 .

Desta forma, é necessário compreender que a saúde e a doença formam um contínuo, em que se relacionam: aspectos econômicos e socioculturais, a experiência pessoal e estilos de vida do ser humano ${ }^{6}$. Portanto, uma vez que um profissional, isoladamente, não consegue dar conta de todas as dimensões do cuidado, são necessárias intervenções cada vez mais complexas no contexto do trabalho em saúde, fazendo-se necessário o reconhecimento da multidimensionalidade do ser humano. (a) Departamento de Clínica e Odontologia Social, Centro de Ciências da Saúde, Universidade Federal da Paraíba (UFPB) Campus Universitário I. João Pessoa, PB, Brasil. 58051-900.fdsforte@ terra.com.br

(b) Discente, Graduação em Odontologia, UFPB. João Pessoa, PB, Brasil. hannah_gil@hotmail.com

(c) Discente, Graduação em Enfermagem, UFPB. João Pessoa, PB, Brasil. shirleybelmonte@ hotmail.com

(d) Discente, Graduação em Farmácia, UFPB. João Pessoa, PB, Brasil. joyce. santtos@hotmail.com

(e) Hospital Universitário Lauro Wanderley. João Pessoa, PB, Brasil.priscilafarias_@ hotmail.com

(f) Departamento de Promoção da Saúde, Centro de Ciências

Médicas, UFPB. João Pessoa, PB, Brasil. socorrotmorais@ hotmail.com

(g) Gerência Executiva de Vigilância em Saúde,

Secretaria Estadual de Saúde da Paraíba. João Pessoa, PB, Brasil. talitha. lira@hotmail.com

(h) Secretaria Estadual de Saúde da Paraíba. João Pessoa, PB, Brasil. mariadefatima.moraes@ gmail.com 
Em 2008, o Ministério da Saúde (MS) e o Ministério da Educação (MEC) lançaram edital convocando as instituições formadoras e as instituições prestadoras de serviços para construírem um projeto que envolvesse professores, preceptores/trabalhadores do Sistema Único de Saúde (SUS) e estudantes de cursos da área da saúde. Esse projeto denominou-se Programa de Educação pelo Trabalho para a Saúde (PET-Saúde). Nos anos seguintes, foram lançados outros editais com temáticas específicas, como o da Vigilância em Saúde e a Saúde Mental. Em 2011, novo edital foi lançado com a proposta de integração do Programa Nacional de Reorientação da Formação Profissional em Saúde (PRO-Saúde), o PET-Saúde e o PRO PET saúde. Já em 2013, foi lançado o edital para os projetos PETSaúde Redes de Atenção à Saúde, do qual este artigo se origina ${ }^{7,8}$.

A articulação entre ensino-serviço-comunidade proposta pelo PET-Saúde Redes de Atenção à Saúde proporciona oportunidades à aprendizagem significativa por meio do sistema tutorial, e fomenta algumas características de natureza coletiva, norteadas pela educação interprofissional. Neste cenário, além dos estudantes da área da saúde, estão os trabalhadores do SUS como preceptores, e os professores como tutores. Os objetivos do grupo tutorial são: o estudo, a reflexão e a crítica sobre uma das redes de atenção à saúde prioritárias para o $\mathrm{MS}^{9-11}$.

Dentro do programa inserido na Universidade Federal da Paraíba (UFPB), em 2013, está o PETRedes Saúde Rede Cegonha (PET-RC). A Rede Cegonha é uma estratégia do MS operacionalizada pelo SUS, fundamentada nos princípios da humanização e da assistência, que assegura, às mulheres, o direito ao planejamento reprodutivo, à atenção humanizada à gravidez, ao parto e ao puerpério; e, às crianças, o direito ao nascimento seguro, ao crescimento e ao desenvolvimento saudável. Tem como objetivos: a implementação de um novo modelo de atenção ao parto, ao nascimento e à saúde da criança; uma rede de atenção que garanta acesso, acolhimento e resolutividade; e a redução das mortalidades materna e neonatal ${ }^{12-14}$.

Este trabalho tem por objetivo relatar as vivências e experiências no PET-RC, ancoradas na formação interprofissional e na prática colaborativa entre estudantes, preceptores e tutores, na promoção do cuidado integral materno-infantil, bem como a contribuição dessas vivências no processo de formação em saúde.

\section{Relato de experiência}

\section{Organização das atividades}

As atividades efetuadas no PET-RC são desenvolvidas em vários cenários de aprendizagem, visando contemplar os diversos pontos da Rede Cegonha do Estado da Paraíba e dos dois municípios envolvidos na proposta. Assim, são cenários localizados na sede da Secretaria Estadual de Saúde da Paraíba (SES-PB); na Gerência-Executiva da Atenção Básica - Saúde da Mulher; na Maternidade Frei Damião; no Hospital Universitário Lauro Wanderley; no Hospital Edson Ramalho; e no Hospital Maternidade de Cabedelo (PB). A seleção desses cenários foi intencional e de comum acordo com a gestão da Rede Cegonha na Paraíba.

A organização operacional do grupo tutorial se deu conforme o edital, por meio da formação de seis subgrupos, contendo, cada um, dois estudantes e um preceptor, sob a supervisão de um tutor. Os estudantes envolvidos fazem os seguintes cursos: Enfermagem, Farmácia, Fonoaudiologia, Medicina, Nutrição, Odontologia, Psicologia, Terapia Ocupacional e Serviço Social. A seleção desses estudantes foi pública, com duas etapas consecutivas e complementares, ofertando-se vagas para todos os cursos da área da saúde do Campus I da UFPB. O grupo de preceptores abrange trabalhadores do SUS de Enfermagem e Serviço Social. O trabalho teve início em agosto de 2013 e, após seis meses, optou-se pelo rodízio semestral entre os estudantes, nos diversos cenários, para oportunizar: vivências diferenciadas, melhor compreensão dos diversos pontos da Rede pelos atores envolvidos nesse processo, e fortalecimento das ações e atividades do projeto.

Para Colet ${ }^{15}$, todo projeto compreende uma dimensão cognitiva e uma dimensão pragmática. As discussões das reuniões foram no sentido de apropriação da Rede Cegonha no Estado da Paraíba; 
houve leituras de documentos oficiais da Rede Cegonha, de forma a aproximar os sujeitos envolvidos da rede de atenção à saúde, foco do grupo tutorial. As leituras de artigos e material teórico sobre a Rede Cegonha, sobre saúde da mulher e da criança e sobre as políticas de saúde vigentes dentro desse universo subsidiaram reflexões e discussões, auxiliando a leitura da Rede nos cenários de aprendizagem envolvidos. Ao mesmo tempo, estratégias foram desenvolvidas para que o grupo se fortalecesse, compreendendo seu objetivo, e para que os membros se conhecessem.

O percurso dos estudantes no sistema de saúde da Rede Cegonha deu-se no sentido de oportunizar experiências interprofissionais, ajudando-os a desenvolverem competências e habilidades para a prática colaborativa e produção do cuidado em saúde mais efetivo ${ }^{4,16}$. Ao mesmo tempo, garantiu, ao trabalhador do SUS, o exercício de preceptoria.

Dessa forma, privilegiou-se um movimento, que, conforme destacaram Soubhi et al. ${ }^{17}$, compreendeu a aprendizagem e a prática interprofissional como resultantes da capacidade dos profissionais de estabelecerem um equilibrio dinâmico entre a organização do que eles sabem e o que fazem. No caso do PET-RC, houve outros atores, além dos profissionais: os estudantes e o tutor. Assim, foi preciso compreender e construir pontes para: a colaboração, a identificação dos temas mobilizadores, uma reflexão crítica sobre como resolver os conflitos, planejar e executar coletivamente estratégias de cuidado em saúde.

Esse movimento de aprender com as práticas colaborativas em uma perspectiva usuário-centrada envolve diversas oportunidades de aprendizado: trabalho em equipe, gerenciamento de conflitos, liderença, crítica sobre o processo de trabalho, corresponsabilização e autocrítica. Competências observadas nas diretrizes curriculares dos cursos da saúde. O objetivo não foi o desenvolvimento de competências específicas de um núcleo profissional, mas sim, a promoção da saúde na perspectiva das gestantes.

Nessa perspectiva, o desafio posto não foi a reunião de estudantes e preceptores de núcleos profissionais diferentes da área da saúde. Foi, por meio de vários dispositivos cognitivos (interação de saberes, socialização), pragmáticos (organização do processo de trabalho) e afetivos (subjetividade)2, construir práticas colaborativas em saúde, contribuindo, dessa forma, com a construção da Rede Cegonha nos cenários envolvidos, com base na integralidade e na humanização do cuidado.

Assim, foi importante o respeito à autonomia dos sujeitos envolvidos nesse processo e na proposta de múltiplos olhares dialógicos de diferentes núcleos profissionais sobre os temas mobilizadores.

Do ponto de vista da governança, o PET-RC é um projeto reconhecido pela Universidade Federal da Paraíba em suas instâncias legais, tais como: a Pró-Reitoria de Graduação, a Pró-Reitoria de Extensão, a Pró-Reitoria de Pós-Graduação; e pelos diretores de centros de ensino da Saúde, da Medicina e das Ciências Humanas. Além disso, também foi aprovado pela Comissão Intergestora Bipartite e pelos Conselhos Municipais de Saúde de João Pessoa (PB) e Cabedelo (PB).

Isso possibilitou dispositivos e arranjos institucionais para a execução de ações interprofissionais e práticas colaborativas nos cenários do PET-RC, bem como autonomia para o planejamento dessas ações e para o entendimento, por parte da gestão acadêmica e dos serviços de saúde, do envolvimento de diversos cenários na proposta do grupo tutorial.

Colet $^{15}$ destacou a necessidade de um enquadramento institucional dos projetos interprofissionais, o que ofereceria condições adequadas para sua execução, sendo este um dos indicadores de avaliação.

Desde sua criação, a UFPB desenvolve projetos de extensão e pesquisa, sendo uma importante instituição de formação e pesquisa no Estado da Paraíba. Participou de todos os projetos de reorientação da formação e, ao longo dos anos, desde a criação da Secretaria de Gestão do Trabalho e Educação na Saúde, do MS, em 2003, vem construindo uma relação dialógica com os municipios onde está inserida e, também, com o governo do Estado da Paraíba. Os cursos da saúde passaram por reformas em suas propostas pedagógicas, aproximando-se, cada vez mais, dos serviços de saúde.

Por outro lado, a rede de atenção à saúde vem se organizando para receber os estudantes, de modo a fortalecer a formação, com uma proposta de Rede Escola, fortalecendo, também, a integralidade do cuidado em saúde. Assim, foram selecionados pontos estratégicos da Rede Cegonha, tanto da atenção como da gestão, no Estado da Paraíba, para favorecer a governabilidade do projeto, no que diz respeito à educação interprofissional e à atenção à saúde das mulheres. 
Reconhece-se que a Rede Cegonha, no Estado da Paraíba, está em construção. A rede de cuidados da saúde da mulher e da criança estava com dificuldades de gerenciar os serviços. Assim, como o gestor e os trabalhadores do SUS envolvidos na Rede, o grupo PET-Rede Cegonha foi desafiado a refletir sobre a Rede em João Pessoa (PB) e Cabedelo (PB).

O estímulo a práticas interprofissionais é uma proposta de aprendizado para o enfrentamento de um sistema de saúde fragmentado. Pois, estando coeso e em diálogo, em um movimento de construção coletiva, o grupo tutorial pode compreender sobre a potencialidade do trabalho em equipe para a construção do projeto terapêutico singular de cada sujeito (gestante, mãe e criança), ofertando serviço de melhor qualidade.

O PET-RC nesse cenário é privilegiado, pois a própria formação do grupo favorece ao aprendizado colaborativo. Assim, o setor educação (aquele que oferta a formação) e o prestador de serviços de saúde, ao dialogarem, estão oportunizando, aos atores envolvidos, um espaço de troca, reflexão, crítica, construção e aprendizado. As discussões, planejamento, execução das atividades e a avaliação permitiram a educação interprofissional e a prática colaborativa dos sujeitos envolvidos.

\section{A formação em saúde e a interprofissionalidade}

A abordagem pedagógica adotada no grupo tutorial foi a metodologia ativa, com ênfase na problematização do cotidiano dos serviços de saúde da Rede Cegonha. Batista ${ }^{16}$ destacou a importância da aprendizagem de jovens e adultos, a partir da: aprendizagem significativa, aprendizagem baseada nas interações e a aprendizagem baseada na prática como pressuposto da educação interprofissional.

No caso do PET-RC, esse processo visa oportunizar, aos estudantes, preceptores e tutor, o estímulo ao desenvolvimento de novas práticas do trabalho em saúde e do aprender em saúde, com proposta pedagógica embasada nos princípios da educação interprofissional. Assim, para a formação dos subgrupos, privilegiaram-se estudantes de diferentes cursos. As ações em campo tinham o objetivo de contribuir para processos de ensino/aprendizagem alicerçados na observação, na reflexão e crítica na identificação de problemas do cotidiano e na perspectiva de construção coletiva para o enfrentamento das questões levantadas.

O planejamento das atividades ocorreu de forma coletiva e articulada com todos os integrantes do grupo por meio de reuniões semanais, entendidas como espaço de partilha, troca e escuta. Para isso, no início das atividades, estabeleceram-se prioridades com base nas necessidades identificadas pelos estudantes e preceptores nos campos de atuação.

O mecanismo utilizado nas reuniões foram o encontro e o diálogo. Valores permearam todo o trabalho, sejam nas reuniões ou no trabalho nos cenários: éticos, respeito mútuo, solidariedade, corresponsabilização, direito e equidade.

No documento da OMS 18 "Framework for action on interprofissional education e collaborative practice" é destacado que a educação e a prática da colaboração interprofissional são uma estratégia inovadora de formação em saúde, que, se usada, desempenha papel importante para melhorias na formação.

Assim sendo, o conhecimento gerado foi numa perspectiva problematizadora, buscando inserir o estudante nessa realidade, bem como para a compreensão do processo de trabalho dos preceptores e demais trabalhadores do SUS, fazendo uma reflexão entre a teoria e a prática.

Os atores envolvidos nesse processo estão inseridos como protagonistas do processo ensino/ aprendizagem, e as discussões geradoras de produtos contribuem para a construção da Rede Cegonha e fortalecimento do SUS, na perspectiva da integralidade do cuidado e da educação interprofissional. Assim, o formato do grupo tutorial é plural no sentido da origem de seus membros e singular no sentido da construção de sua identidade e processo de trabalho. O grupo tutorial do PET-RC permitiu que não só estudantes de diferentes cursos interagissem, mas, também, profissionais de diferentes núcleos profissionais e de pontos distintos da Rede Cegonha conversassem. Assim, vislumbraram-se caminhos não só para uma formação contextualizada, mas, também, para transformação e qualificação das práticas de saúde e para a organização das ações e dos serviços. 
A educação interprofissional e a prática colaborativa no grupo tutorial e nas ações no território proporcionaram aproximação com a realidade, e também fizeram com que muitos dispositivos usados estimulassem o trabalho em equipe de forma colaborativa, nos diversos cenários envolvidos. Pois, compreende-se que a atuação conjunta não significa a prática colaborativa.

\section{PET-Rede Cegonha no território}

Nos cenários de aprendizagem, o grupo PET-RC fez um movimento de propor novas ações ou uma reorganização do processo de trabalho dos profissionais de saúde. O trabalho em equipe será bemsucedido se houver interação entre os sujeitos envolvidos no processo, de modo que competências, habilidades e conhecimentos diferentes atuem de forma dialogada e partilhada, na perspectiva de práticas colaborativas.

A experiência demonstrou que a educação interprofissional proporcionou a promoção da saúde das gestantes nas maternidades envolvidas, no que diz respeito à discussão sobre direito, saúde e cidadania, segurança do paciente, e ao trabalho mais integrado nas equipes envolvidas, estendendo algumas ações para além do grupo PET-RC. Nesse sentido, os profissionais de saúde envolvidos sentiram necessidade de revisitar suas práticas e saberes, reconhecendo as ações a serem desenvolvidas e aproximando-se dos conceitos estudados.

Essas ações ampliaram o trabalho das equipes no sentido de promover a educação em saúde com as mulheres e seus acompanhantes, bem como a organização do trabalho em equipe e as ações interprofissionais colaborativas, visando à integralidade e à resolutividade das ações, e à tomada das decisões. Embora se tenha avançado muito, ainda há muito a ser feito.

Nas maternidades, foram desenvolvidas ações na recepção por meio: do acolhimento com classificação de risco, pré-parto, sala de parto e enfermarias. As ações permearam, sobretudo, a educação em saúde, com ênfase na prevenção e na promoção, por intermédio do contato direto com as gestantes, as puérperas e os recém-nascidos. Um fator facilitador no grupo PET-RC foi a partilha de objetivo comum aos atores envolvidos, e todos estavam cientes de seus papéis no grupo².

Entre as estratégias utilizadas, podem-se destacar as rodas de conversa realizadas nas enfermarias e o grupo de gestantes, realizados mensalmente, nos quais foram abordados temas diferentes, sempre valorizando o saber da gestante, da puérpera e dos acompanhantes, trazendo reflexão e diálogo entre os atores envolvidos. Essas conversas permitiram, à equipe de trabalhadores do SUS, aos preceptores e aos estudantes do PET envolvidos, o conhecimento do cotidiano e dos hábitos das famílias, permitindo-Ihes atuações diretas nos contextos familiares.

O grupo de gestantes de uma das maternidades é pautado nas práticas educativas sob a perspectiva da educação popular e tem como público-alvo as gestantes que realizam pré-natal de alto risco. Os encontros ocorrem uma vez ao mês, e os assuntos da roda de conversa são oriundos da comunidade de gestantes e acompanhantes. Por ser uma roda de conversa, o assunto central é debatido, o que torna possível o diálogo sobre outros temas relevantes e de interesse do público-alvo. Isto ocorre em função da relação que vai se estabelecendo no grupo, priorizando a humanização, o acolhimento, a escuta, e oportunizando o diálogo entre a comunidade e a equipe de trabalho, com vistas à autonomia dos sujeitos envolvidos. Enfim, as rodas permitiram que dúvidas, mitos, impressões, percepções e conceitos fossem expostos e discutidos, de maneira simples, utilizando-se uma linguagem acessível.

Outra questão muito importante nas rodas de conversa do grupo de gestantes foi a valorização da experiência própria em relação ao assunto tratado, o qual, geralmente, teria sido vivenciado em gestações anteriores e passava a ser compartilhado não só com as gestantes experientes, mas, também, com as que estavam na primeira gestação.

Uma estratégia adotada nesta experiência foi o aprendizado por meio de ações educativas em saúde, entendendo-as não como meros repasses de informação, mas como práxis que buscam gerar vínculos, significados e aprendizados. Além disso, estar na posição de facilitar a aprendizagem já é, em si, uma aprendizagem, na qual o próprio indivíduo - que se coloca ou é colocado como educador também vive e se transforma em educando ${ }^{19}$. 
O uso de práticas educativas visa fortalecer o conhecimento do usuário, percebendo a saúde não só como resultado de práticas individuais, mas, também, como reflexo das condições de vida em geral ${ }^{20}$. Sendo assim, a educação em saúde pode ser uma ferramenta de atuação em saúde por possibilitar a troca entre o conhecimento técnico e o popular, permitindo o desenvolvimento de ações de prevenção e promoção de saúde.

Nesse contexto de práticas educativas, temos a influência da Educação Popular. A realização de ações na perspectiva da Educação Popular possibilita a participação ativa dos envolvidos, valorizando o diálogo e o desenvolvimento da autonomia. A Educação Popular em Saúde realiza ações que envolvem as dimensões do diálogo, do respeito e da valorização do saber popular, sendo considerada como um instrumento de construção para uma saúde mais integral e adequada à vida da população ${ }^{21}$.

Partindo da pedagogia dialógica de Paulo Freire ${ }^{22}$, o trabalho em grupo desperta, nas pessoas, a sua autonomia e favorece o reconhecimento de sujeitos portadores de saberes sobre o processo saúdedoença-cuidado, e de condições concretas de vida.

A educação em saúde na perspectiva da Educação Popular se configura como uma importante ferramenta para a promoção da saúde, tanto no âmbito individual quanto no familiar e comunitário, na prevenção dos principais agravos à saúde. Ela proporciona a interação entre a teoria e a prática, assim como entre o saber científico e o popular, permitindo uma aprendizagem interativa e dinâmica. Além de maior interação entre os participantes e maior compreensão, bem como novos aprendizados e afetos, possibilitando mais engajamento, responsabilização e motivação ${ }^{23-26}$. O PET-RC proporcionou uma maior integração entre o serviço, o ensino e a comunidade, possibilitando, assim, a atuação com ênfase nas particularidades e necessidades do público-alvo.

Na maternidade, observou-se a potencialidade do trabalho em equipe, desde a identificação das demandas ao planejamento compartilhado das atividades a serem desenvolvidas, de forma que houvesse a distribuição de responsabilidades entre os trabalhadores do SUS, aqueles trabalhadores preceptores do PET-RC e os estudantes bolsistas. Nesse movimento de pactuação coletiva, o diálogo foi sempre necessário para exposição de ideias, possibilidades, limites e dificuldades a serem enfrentadas. À medida que as ações eram executadas, foi necessária, muitas vezes, a ressignificação de estratégias, como ponto de partida para as ações seguintes. Observou-se, ao longo da realização dos grupos de gestantes, uma socialização de saberes e uma discussão da Rede Cegonha enquanto política do cuidado integral à saúde materno-infantil. O objetivo desse exercício de planejamento, execução e avaliação coletivo foi desenvolver a competência para o trabalho interprofissional.

Outro cenário se deu na Divisão Saúde da Mulher, da Gerência Executiva da Atenção Básica, da Secretaria Estadual de Saúde do Estado da Paraíba. Os estudantes participaram das discussões do grupo condutor da Rede Cegonha do Estado da Paraíba, que foi instituído em 2012. Esse colegiado gestor é composto por representações: das secretarias municipais e estadual, das maternidades, dos conselhos municipais e estadual, além dos apoiadores do MS, e tem por objetivo a implementação da Rede Cegonha para o cuidado à gestante, à puérpera e às crianças.

Nessas reuniões, os estudantes observaram a discussão das dificuldades e limitações, dos entraves burocráticos e financeiros, das fragilidades da rede e dos desafios a serem superados - como a falta de comunicação entre os serviços e a gestão -, em busca de soluções capazes de transformar as situações encontradas ou reveladas.

Nesse ínterim, o grupo composto por estudantes, preceptor e profissionais era, constantemente, desafiado a desenvolver novas práticas e ações em saúde, pautadas por conceitos como: integralidade, interprofissionalidade e promoção da saúde.

Foram propostas pesquisas voltadas para as necessidades dos serviços e da população, que funcionaram como eixos estruturadores de busca pelo conhecimento, tanto para os estudantes como para os profissionais de saúde envolvidos.

A avaliação foi contínua e formativa, buscando compreender os avanços e os desafios de cada ator nesse processo. O diálogo como ponto de partida e a autoavaliação também foram privilegiados para que a subjetividade de cada ator viesse à tona. Utilizou-se o diário de campo para o registro das vivências significativas, para o levantamento de questões importantes e a definição de prioridades, além do registro de impressões e questionamentos. O diário teve, ainda, o objetivo de aprimorar as 
atividades e as estratégias adotadas.

A dimensão subjetiva da interprofissionalidade foi explicada por Soubhi et al. ${ }^{17}$ como atitudes e crenças dominantes que podem favorecer as relações entre os profissionais, como, também, uma predisposição dos atores envolvidos a saírem de suas zonas de conforto, no que diz respeito às práticas e às fronteiras disciplinares.

Espera-se que essas vivências sejam capazes de preparar melhor os futuros profissionais de saúde para atuarem dentro dos princípios estruturadores do SUS, e que a qualificação profissional implique, também, a elevação da qualidade da atenção oferecida à população, contribuindo para o desenvolvimento do senso de responsabilidade social nos estudantes da área da saúde.

\section{Considerações finais}

A inserção dos acadêmicos no serviço tem demonstrado ser uma excelente oportunidade para conhecer o funcionamento e a realidade do SUS, viabilizando uma maior integração da teoria com a prática - inclusive, com as práticas colaborativas - e a interprofissionalidade. Entende-se que programas como o PET-Saúde vêm contribuir para o aprimoramento dos profissionais da saúde, pois a relação entre ensino e serviço tem proporcionado uma formação diferenciada aos acadêmicos, que passam a vivenciar os desafios da materialização do SUS e estimulam, assim, uma visão crítica para a rede de serviços no sistema. Nesse mesmo movimento, a vivência do grupo tutorial permite aos preceptores desenvolverem competências para o trabalho interprofissional e as práticas colaborativas, além do exercício de preceptoria.

Percebemos, ainda, a necessidade de se reafirmar uma atuação interprofissional com foco na integralidade do cuidado, o que permitiria um trabalho com objetivos de prevenção e promoção da saúde materno-infantil, a partir da compreensão ampliada do processo saúde-doença.

Cada vez mais, se coloca em pauta a necessidade da integração entre os diversos profissionais, para que estes possam inventar e reinventar formas de atuar interdisciplinarmente, proporcionando melhores resultados ao trabalho.

Portanto, faz-se necessário que as oportunidades de formação nessa linha sejam multiplicadas, para atender às demandas sociais crescentes, contribuindo na construção de um modelo assistencial pautado nos princípios do SUS, que venham a fortalecer a promoção ao cuidado integral e possibilitar que os profissionais saibam atuar em equipe, ofertando serviços resolutos e de qualidade para a população.

A partir das reuniões para discussão, planejamento, relato e avaliação, fortaleceu-se a integração de saberes e fazeres em saúde de profissionais de diversos pontos da Rede Cegonha no Estado da Paraíba. Ao mesmo tempo, no cotidiano dos serviços, os estudantes desenvolveram atividades com base na Educação Popular em Saúde, na perspectiva da integralidade do cuidado, como, também, puderam observar o processo de trabalho em saúde, no que se refere às dificuldades, às fragilidades, às potencialidades e à rotina dos serviços de saúde, sejam elas do campo da gestão e/ou da atenção. As vivências oportunizaram as práticas colaborativas em saúde e permitiram o diálogo entre os atores envolvidos.

\section{Colaboradores}

Franklin Delano Soares Forte e Hannah Gil de Farias Morais trabalharam na concepção, escrita e revisão crítica do conteúdo do artigo. Shirley Arruda Guimarães Rodrigues, Joyce da Silva Santos e Priscila Farias de Albuquerque Oliveira responsabilizaram-se pela análise da discussão e escrita do artigo. Maria do Socorro Trindade Morais, Talitha Emanuelle Barbosa Galdino de Lira e Maria de Fátima Moraes Carvalho trabalharam na redação e revisão crítica. 


\section{Referências}

1. Barr H, Koppel I, Hamminck M, Freeth DS. Effective interprofissional education: assumption, argument and evidence. Oxford: Wikey Blackwell; 2005.

2. Ellery AEL. Interprofissionalidade na estratégia saúde da família: condições de possibilidade para a integração de saberes e a colaboração interprofissional [tese]. Fortaleza (CE): Universidade Federal do Ceará; 2012.

3. Hammick $M$, Zwarestein $M$, Reeves $S$, Barr H, Goldman J, Freeth $D$, et al. Interprofessional education: effects on professional practice and health outcomes. $3 \mathrm{a}$ ed. Toronto: Cochrane Database of Systematic Reviews; 2000.

4. World Health Organization. Marco para ação em educação interprofissional e prática colaborativa. Genebra: World Health Organization; 2010.

5. Vilela EM, Mendes IJM. Interdisciplinaridade e saúde: estudo bibliográfico. Rev LatinoAm Enfermagem. 2003; 11(4):525-31.

6. Cardoso JP, Vilela ABA, Souza NR. Formação interdisciplinar: efetivando propostas de promoção da saúde no SUS. Rev Bras Promoc Saude. 2007; 20(4):252-8.

7. Haddad AE, Brenelli SL, Cury GC, Puccini RF, Martins MA, Ferreira JR, et al. Pró-Saúde e PET-Saúde: a construção da política brasileira de reorientação da formação profissional em Saúde. Rev Bras Educ Med. 2012; 36 Supl 2:3-4.

8. Ferreira JR, Haddad AE, Brenelli SL. Pró-Saúde e PET-Saúde: experiências exitosas de integração ensino-serviço. Rev Bras Educ Med. 2012; 36 Supl 1:3-4.

9. Costa MV, Patrício KP, Câmara AM, Azevedo GD, Batista SH. Pró-Saúde e PET-Saúde como espaços de educação interprofissional. Interface (Botucatu). 2015; 19(1):709-20.

10. Camara AM, Grosseman S, Pinho DL. Educação interprofissional no Programa PETSaúde: a percepção de tutores. Interface. 2015; 19(1):817-29.

11. Batista SH, Jansen B, Assis EQ, Senna MIB, Cury GC. Formação em Saúde: reflexões a partir dos Programas Pró-Saúde e PET-Saúde. Interface (Botucatu). 2015; 19(1):743-52.

12. Ministério da Saúde (BR). Portaria no 1.459, de 24 de junho de 2011. Institui, no âmbito do Sistema Único de Saúde - SUS - a Rede Cegonha. Brasília (DF): Ministério da Saúde; 2011.

13. Ministério da Saúde (BR). Portaria n 650, de 5 de outubro de 2011. Dispõe sobre os Planos de Ação Regional (PAR). Brasília (DF): Ministério da Saúde; 2011.

14. Ministério da Saúde (BR). Ministério da Educação. Secretaria de Atenção à Saúde. Manual Prático para implementação da Rede Cegonha. Brasília (DF): Ministério da Saúde; 2011.

15. Colet NR. Enseignement universitaire et interdisciplinarité: um cadre pour analyser, agir et évalue. Bruxelles: Boeck \& Larcier; 2002.

16. Batista NA. Educação interprofissional em Saúde: concepções e práticas. Cad FNEPAS. 2012; 2(1):25-8.

17. Soubhi H, Colet NR, Gilbert JHV, Lebel P, Thivierge RL, Hudon C, et al. Interprofissional learning in the trenches: fotering collective capability. J Interprof Care. 2009; 23(1):52-7.

18. World Health Organization. Framework for action on interprofessional education and collaborative practice. Genebra: World Health Organization; 2010.

19. Sampaio J, Santos GC, Agostini M, Salvador AS. Limites e potencialidades das rodas de conversa no cuidado em saúde: uma experiência com jovens no sertão pernambucano. Interface (Botucatu). 2014; 18(2):1299-311. 
20. Traesel CA, Bedin FNP, Gallina LS, Daneluz SFC, Santos AC, Melo TT, et al. Educação em saúde: fortalecendo a autonomização do usuário. São Paulo: Hucitec; 2004.

21. Vasconcelos EM. Educação popular e a atenção à saúde da família. 3a ed. São Paulo: Hucitec; 2006.

22. Freire P. Pedagogia da autonomia: saberes necessários à prática educativa. 31a ed. São Paulo: Paz e Terra; 2005.

23. Hall P. Interprofessional teamwork: professional cultures as barriers. J Interprof Care. 2005; 19(1):188-96.

24. Interprofessional Education Collaborative Expert Panel. Core competencies for interprofessional collaborative practice: Report of an expert panel. Washington (DC): Interprofessional Education Collaborative; 2011.

25. Leite MF, Ribeiro, KSQS, Anjos UU, Batista PSS. Extensão popular na formação profissional em saúde para o SUS: refletindo uma experiência. Interface (Botucatu). 2014; 18(2):1569-78.

26. Passos E, Carvalho YM. A formação para o SUS abrindo caminhos para a produção do comum. Saude Soc. 2015; 24(1):92-101. 
A educação interprofissional é uma possibilidade de capacitação de estudantes de diferentes cursos da área da saúde, na perspectiva do aprendizado colaborativo, bem como do trabalho em equipe e em rede, visando à integralidade do cuidado em saúde. Este trabalho tem por objetivo relatar as vivências e experiências no Programa de Educação pelo Trabalho para a Saúde/Rede Cegonha, com ênfase na educação interprofissional e nas práticas colaborativas em saúde para a promoção do cuidado integral materno-infantil, bem como na contribuição dessas vivências durante o processo de formação em saúde.

Palavras-chave: Trabalho em Saúde. Formação interprofissional. Rede Cegonha.

\section{Interprofessional education and the Education through Work for Health Program "Stork Network": leveraging changes in education}

The interprofessional education is the opportunity to train students from different courses in the health area under the perspective of collaborative learning, as well as in teamwork and networking, aiming at the comprehensiveness of health care. This paper aims to report experiences in Education through Work for Health Program "Stork Network", focusing on interprofessional education and collaborative practice in health to promote maternal and child comprehensive care, as well as the contribution of these experiences during the health education process.

Keywords: Work in Health. Interprofessional education. Stork network.

\section{Educación interprofesional y Programa de Educación para el trabajo en Salud/Red Cigüeña: potencializando cambios en la formación académica}

La educación interprofesional es una posibilidad para la formación de estudiantes de diferentes cursos en el área de la salud, a partir de la perspectiva del aprendizaje colaborativo, trabajo en equipo y en red, dirigidos a la integridad del cuidado en salud. Este trabajo tiene como objetivo informar sobre las experiencias y vivencias en el programa PET Salud/Red Cigüeña, centrándose en la educación interprofesional y la práctica de colaboración en materia de salud para promover la atención integral maternoinfantil, así como aportar material relativo a la contribución de estas experiencias durante el proceso de formación en salud.

Palabras clave: Trabajo en Salud. Formación interprofesional. Red Cigüeña. 